\title{
TERMINATION OF REWRITING WITH RIGHT-FLAT RULES MODULO PERMUTATIVE THEORIES *
}

\author{
LUIS BARGUNOÓ $^{a}$, GUILLEM GODOY $^{b}$, EDUARD HUNTINGFORD, AND ASHISH TIWARI ${ }^{c}$ \\ ${ }_{a, b, c}$ Universitat Politècnica de Catalunya, Jordi Girona 1, Barcelona, Spain \\ e-mail address: luisbargu@gmail.com, ggodoy@lsi.upc.edu, eduard.hl@gmail.com \\ ${ }^{c}$ SRI International, Menlo Park, CA 94205 \\ e-mail address: tiwari@csl.sri.com
}

\begin{abstract}
We present decidability results for termination of classes of term rewriting systems modulo permutative theories. Termination and innermost termination modulo permutative theories are shown to be decidable for term rewrite systems (TRS) whose right-hand side terms are restricted to be shallow (variables occur at depth at most one) and linear (each variable occurs at most once). Innermost termination modulo permutative theories is also shown to be decidable for shallow TRS. We first show that a shallow TRS can be transformed into a flat (only variables and constants occur at depth one) TRS while preserving termination and innermost termination. The decidability results are then proved by showing that (a) for right-flat right-linear (flat) TRS, non-termination (respectively, innermost non-termination) implies non-termination starting from flat terms, and (b) for right-flat TRS, the existence of non-terminating derivations starting from a given term is decidable. On the negative side, we show PSPACE-hardness of termination and innermost termination for shallow right-linear TRS, and undecidability of termination for flat TRS.
\end{abstract}

\section{INTRODUCTION}

Termination is an important property of computing systems and it has generated significant renewed interest in recent years. There has been progress on both the theoretical and practical aspects of proving termination of many different computing paradigms - such as term rewrite systems (TRS), functional programs, and imperative programs. Innermost termination refers to termination of rewriting restricted to the innermost strategy, which forces the complete evaluation of all the subterms before a rule is applied at any position.

1998 ACM Subject Classification: F.4.2.

Key words and phrases: term rewriting, termination, decidability, complexity.

* A preliminary version GHT07] containing some of the results appeared in the Proceedings of the 18th International Conference on Rewriting Techniques and Applications, RTA 2007.

${ }^{a}$ Supported by Spanish Ministry of Education and Science by the FORMALISM project (TIN2007-66523).

${ }^{b}$ Supported by Spanish Ministry of Education and Science by the FORMALISM project (TIN2007-66523) and the LOGICTOOLS-2 project (TIN2007-68093-C02-01).

${ }^{c}$ Supported in part by the National Science Foundation under grants CNS-0720721 and CSR-0917398.

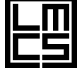

DOI:10.2168/LMCS-6 (3:8) 2010
(C) L. Barguñó, G. Godoy, E. Huntingford, and A. Tiwari (c) Creative Commons 
It corresponds to the "call by value" computation of programming languages. A typical example of a TRS that is innermost terminating but not terminating is the following [Toy87]:

$$
\{f(0,1, x) \rightarrow f(x, x, x), c \rightarrow 0, c \rightarrow 1\}
$$

The non-terminating derivation

$$
\underline{f(0,1, c)} \rightarrow f(\underline{c}, \underline{c}, c) \rightarrow^{2} f(0,1, c) \rightarrow \cdots
$$

is not possible with innermost rewriting, since $c$ has to be normalized before a rule can be applied at the root position to reduce $f(0,1, c)$.

Often, a term rewrite system contains rules that are trivially non-terminating (like commutativity: $f(x, y) \rightarrow f(y, x))$ and one desires to ensure a weaker notion than termination, namely termination of a term rewrite system $R$ modulo a theory $E$ (for example, when $E=\{f(x, y) \rightarrow f(y, x)\})$. Althought $R \cup E$ could be non-terminating, in some cases the important question is to determine if there is a derivation with $R \cup E$ that has infinitely many rewrite steps with rules in $R$.

While termination is undecidable for general TRS and string rewrite systems [HL78], several subclasses with decidable termination problem have been identified. Termination is decidable for ground TRS [HL78]; in fact, in polynomial time [Pla93]. Termination is decidable for right-ground TRS [Der81] and also for the more general class that also has collapsing (right-variable) rules [GT04]. Later, it was shown that termination is decidable for TRS that contain any combination of right-ground, collapsing, and shallow right-linear rewrite rules [GT05]. There are further known decidability results about shallow left-linear and shallow right-linear TRS [WS06].

This paper focuses on termination and innermost termination of TRS for rewriting modulo permutative theories. Here we extend the results of our conference paper [GHT07] by generalizing from plain rewriting to rewriting modulo permutative theories. Moreover, we provide extended proofs of our earlier results, and a new PSPACE-hardness result.

The main contributions of the paper are as follows:

(1) In Section 4, we prove that termination starting from a given fixed term is decidable for right-shallow TRS and rewriting modulo permutative theories. This result is used to obtain subsequent results.

(2) In Section 5, we consider innermost rewriting modulo permutative theories and show that termination is decidable for shallow TRS.

(3) In Section 6, we show that termination (and innermost termination as well) is decidable for rewriting modulo permutative theories using TRS whose right-hand side terms are both shallow and linear. There is no restriction on the left-hand side terms. Thus, right-ground TRS and shallow right-linear TRS are both contained in our class.

(4) In Section 7, we prove that termination, as well as innermost termination, is PSPACEhard for flat (and hence shallow) right-linear TRS.

(5) In Section 8, we show undecidability of termination for flat TRS and plain rewriting, and undecidability of termination for right-shallow TRS and innermost rewriting.

Uchiyama, Sakai and Sakabe [USS10] have recently also generalized the results of our conference paper [GHT07] by replacing syntactic restrictions on the rewrite rules by syntactic restrictions on the dependency pairs. Specifically, termination and innermost termination were shown to be decidable for TRS whose dependency pairs are right-linear and rightshallow; and innermost termination was shown to be decidable for TRS whose dependency pairs are shallow. 


\section{Preliminaries}

We use standard notation from the term rewriting literature [BN98]. A signature $\Sigma$ is a (finite) set of function symbols with arity, which is partitioned as $\cup_{i} \Sigma_{i}$ such that $f \in \Sigma_{m}$ if the arity of $f$ is $m$. Symbols in $\Sigma_{0}$, called constants, are denoted by $a, b, c, d, e$, with possible subscripts. The elements of a set $\mathcal{X}$ of variable symbols are denoted by $x, y, z$ with possible subscripts. The set $\mathcal{T}(\Sigma, \mathcal{X})$ of terms over $\Sigma$ and $\mathcal{X}$, is the smallest set containing $\mathcal{X}$ and such that $f\left(t_{1}, \ldots, t_{m}\right)$ is in $\mathcal{T}(\Sigma, \mathcal{X})$ whenever $f \in \Sigma_{m}$, and $t_{1}, \ldots, t_{m} \in$ $\mathcal{T}(\Sigma, \mathcal{X})$. A position is a sequence of positive integers. The set of positions of a term $t$, denoted $\operatorname{Pos}(t)$, is defined recursively as follows. If $t$ is a variable then $\operatorname{Pos}(t)$ is $\{\lambda\}$, where $\lambda$ represents the empty sequence. If $t$ is of the form $f\left(t_{1}, \ldots, t_{m}\right)$, then $\operatorname{Pos}(t)$ is $\{\lambda\} \cup\left\{1 . p \mid p \in \operatorname{Pos}\left(t_{1}\right)\right\} \cup \ldots \cup\left\{m . p \mid p \in \operatorname{Pos}\left(t_{m}\right)\right\}$. If $p$ is a position and $t$ is a term, then by $\left.t\right|_{p}$ we denote the subterm of $t$ at position $p$ : we have $\left.t\right|_{\lambda}=t$ (where $\lambda$ denotes the empty sequence) and $\left.f\left(t_{1}, \ldots, t_{m}\right)\right|_{i . p}=\left.t_{i}\right|_{p}$ if $1 \leq i \leq m$ (and is undefined if $i>m$ ). By $|p|$ we denote the length of a position $p$. We also write $t[s]_{p}$ to denote the term obtained by replacing in $t$ the subterm at position $p$ by the term $s$. More formally, $t[s]_{\lambda}$ is $s$, and $f\left(t_{1}, \ldots, t_{i-1}, t_{i}, t_{i+1}, \ldots, t_{m}\right)[s]_{i . p}$ is $f\left(t_{1}, \ldots, t_{i-1}, t_{i}[s]_{p}, t_{i+1}, \ldots, t_{m}\right)$. For example, if $t$ is $f(a, g(b, h(c)), d)$, then $\left.t\right|_{2.2 .1}=c$, and $t[d]_{2.2}=f(a, g(b, d), d)$. Note that $s=s\left[\left.s\right|_{p}\right]_{p}$, and that the equality $s=s[u]_{p}$ implies $\left.s\right|_{p}=u$. The set of all subterms of a term $s$ is denoted by $\operatorname{Subterms}(s)$. The symbol occurring at the root of a term $t$ is denoted as $\operatorname{root}(t)$. We write $p_{1}>p_{2}$ (equivalently, $p_{2}<p_{1}$ ) and say $p_{1}$ is below $p_{2}$ (equivalently, $p_{2}$ is above $p_{1}$ ) if $p_{2}$ is a proper prefix of $p_{1}$, that is, $p_{1}=p_{2} \cdot p_{2}^{\prime}$ for some non-empty $p_{2}^{\prime}$. In this case, by $p_{1}-p_{2}$ we denote $p_{2}^{\prime}$. By $p_{1} \leq p_{2}$ we denote that either $p_{1}<p_{2}$ or $p_{1}=p_{2}$ hold. Positions $p$ and $q$ are parallel, denoted $p \| q$, if $p \nsupseteq q$ and $q \nsupseteq p$ hold. By $\mathcal{V}(t)$ we denote the set of all variables occurring in a term $t$. The height of a term $s$ is 0 if $s$ is a variable or a constant, and $1+\operatorname{Max}_{i}\left(\right.$ height $\left.\left(s_{i}\right)\right)$ if $s=f\left(s_{1}, \ldots, s_{m}\right)$. The depth of an occurrence at position $p$ of a term $t$ in a term $s=s[t]_{p}$ is $|p|$. Sometimes we will denote a term $f(t)$ by the simplified form $f t$ when the arity of $f$ is 1 , and $t[s]_{p}$ by $t[s]$ when $p$ is clear from the context or not important.

A substitution $\sigma$ is a mapping from variables to terms. It can be homomorphically extended to a function from terms to terms: $\sigma(t)$ denotes the result of simultaneously replacing in $t$ every $x \in \operatorname{Dom}(\sigma)$ by $\sigma(x)$. For example, if $\sigma$ is $\{x \mapsto f(b, y), y \mapsto a\}$, then $\sigma(g(x, y))$ is $g(f(b, y), a)$.

A rewrite rule over $\Sigma$ is a pair of terms $(l, r)$ of $\mathcal{T}(\Sigma, \mathcal{X})$, denoted by $l \rightarrow r$, with left-hand side $l$ and right-hand side $r$. We make the usual assumptions for the rules, i.e. $l$ is not a variable, and all variables occurring in the term $r$ also occur in the term $l$. A term rewrite system (TRS) $R$ over $\Sigma$ is a finite set of rewrite rules over $\Sigma$. We often assume $\Sigma$ as implicit when talking about a TRS $R$. We say that $s$ rewrites to $t$ in one step at position $p$ (by $R$ ), denoted by $s \rightarrow_{R, p} t$, if $\left.s\right|_{p}=\sigma(l)$ and $t=s[\sigma(r)]_{p}$, for some $l \rightarrow r \in R$ and substitution $\sigma$. We also denote such a rewrite step by $\rightarrow_{l \rightarrow r, p, \sigma}$ if we make explicit the used rule $l \rightarrow r$ and substitution $\sigma$. If $p=\lambda$, then the rewrite step $\rightarrow_{R, p}$ is said to be applied at the root. Otherwise, it is denoted by $s \rightarrow_{R,>\lambda} t$.

If $\rightarrow$ is a binary relation on a set $S$, then $\leftrightarrow$ is its symmetric closure, $\rightarrow^{+}$is its transitive closure, $\leftarrow$ is its inverse, and $\rightarrow^{*}$ is its reflexive-transitive closure.

A (rewrite) derivation (from $s$ ) is a sequence of rewrite steps (starting from $s$ ), that is, a sequence $s \rightarrow_{R} s_{1} \rightarrow_{R} s_{2} \rightarrow_{R} \ldots$. With $s \rightarrow_{R}^{*} t$ we denote that $t$ is $R$-reachable from $s$, or a concrete derivation from $s$ to $t$, depending on the context. A term $t$ is context-reachable 
from $s$ with $R$ (with a non-empty context) if there exists a derivation of the form $s \rightarrow_{R}^{*} u$ where $t$ is a (proper) subterm of $u$. The length of a derivation $s \rightarrow_{R}^{*} t$, denoted $\left|s \rightarrow_{R}^{*} t\right|$, is its number of rewrite steps. We denote this derivation as $s \rightarrow_{R}^{0} t, s \rightarrow_{R}^{1} t$ and $s \rightarrow_{R}^{0,1} t$ when this number is 0,1 , and 0 or 1 , respectively. A TRS $R$ is terminating from $s$ if there are no $R$-derivations, $s \rightarrow_{R} s_{1} \rightarrow_{R} \cdots$ with infinitely many rewrite steps. If $R$ is terminating from every term, then $R$ is said to be terminating. A term $s$ is $R$-irreducible (or, in $R$-normal form) if there is no term $t$ such that $s \rightarrow_{R} t$. When there is a unique normal form reachable from a given term $t$ using $R$, it is denoted by $\mathrm{NF}_{R}(t)$. When $R$ is singleton, say $R=\{l \rightarrow r\}$, then $\mathrm{NF}_{R}(t)$ will also be written as $\mathrm{NF}_{l \rightarrow r}(t)$.

A term $t$ is called ground if $t$ contains no variables. It is called shallow if all variable positions in $t$ are at depth 0 or 1 . It is flat if its height is at most 1 . It is linear if every variable occurs at most once.

A rule $l \rightarrow r$ is called ground (flat, shallow, linear) if both $l$ and $r$ are. A rule $l \rightarrow r$ is called left-ground (left-flat, left-shallow, left-linear) if $l$ is. A rule $l \rightarrow r$ is called rightground (right-flat, right-shallow, right-linear) if $r$ is. A rule $l \rightarrow r$ is called collapsing if $r$ is a variable.

A TRS $R$ is called (left-,right-)ground (flat, shallow, linear) if all its rules are. A TRS $R$ is called collapsing if it contains a collapsing rule.

A rewrite step $s \rightarrow_{R, p} t$ is an innermost rewrite step if $\left.s\right|_{p^{\prime}}$ is $R$-irreducible, for all $p^{\prime}>p$. The concepts of reachability and termination can be naturally defined for innermost rewriting.

A set $E$ of pairs of terms is a set of equations if whenever a pair, again written as $l \rightarrow r$, belongs to $E$, the pair $r \rightarrow l$ also belongs to $E$. Given a TRS $R$ and a set of equations $E$, a term $s$ rewrites into a term $t$ with $R$ modulo $E$ in one step, denoted $s \rightarrow_{R / E} t$, if $s \rightarrow_{E}^{*} \rightarrow_{R} \rightarrow_{E}^{*} t$ holds. Note that $s \rightarrow_{R / E}^{+} t$ is equivalent to existence of a derivation of the form $s \rightarrow_{R \cup E}^{*} t$ with at least one rewrite step with $R$. A permutative rule is a linear flat rewrite rule $l \rightarrow r$ satisfying height $(l)=$ height $(r)$ and $\mathcal{V}(l)=\mathcal{V}(r)$. When $E$ contains just permutative rules we say that $E$ is a permutative theory. In the rest of the paper we will always assume that $E$ is a permutative theory defined over the same signature as $R$.

The notion of innermost rewriting is extended to rewriting modulo in the following natural way. A term $s$ is a normal form with respect to $R / E$ if no $R / E$ rewrite step can be applied on $s$. A term $s$ innermost rewrites to $t$ with $R / E$ if there exist terms $u, v$ and a position $p$ satisfying $s \rightarrow_{E}^{*} u \rightarrow_{R, p} v \rightarrow_{E}^{*} t$ and such that any proper subterm of $\left.u\right|_{p}$ is a normal form with respect to $R / E$.

The notion of termination for $R / E$ is naturally defined as the non-existence of a $R / E$ derivation with infinitely many rewrite steps. Note that this is equivalent to the nonexistence of a derivation with $R \cup E$ where infinitely many of the involved steps use $R$.

\section{Flattening and Other Simplifying Assumptions}

In this section we present some standard transformations on the signature and TRS GT05, GHT07, and argue that they preserve termination and innermost termination modulo permutative theories. In particular, we show that we can assume without any loss of generality that

(A1) the signature contains exactly one function symbol with nonzero arity

(A2) all shallow terms are in fact flat.

Readers who believe these claims can jump to the next section. 
The discussion is written for general termination, but it is also valid when we interpret termination as innermost termination. To this end, in the innermost case we assume that for a given TRS, all the rules $l \rightarrow r$ such that $l$ has a proper subterm that is not a normal form have been removed. Note that these rules can not be used in an innermost derivation. Thus, when considering innermost rewriting, we assume that (A3) if $l \rightarrow r$ is a rule in $R$, then all proper subterms of $l$ are in normal form.

We will always assume that all terms are constructed over a given fixed signature $\Sigma$ that contains several constants and only one non-constant function symbol $f$. If this was not the case, we can define a transformation $T$ from terms over $\Sigma$ into terms over a new signature $\Sigma^{\prime}$ as follows. Let $m$ be the maximum arity of a symbol in $\Sigma$ plus 1 . We choose a new function symbol $f$ with arity $m$ and define the new signature $\Sigma^{\prime}=\Sigma_{0}^{\prime} \cup \Sigma_{m}^{\prime}$ as $\Sigma_{0}^{\prime}=\Sigma$ and $\Sigma_{m}^{\prime}=\{f\}$. Note that all symbols of $\Sigma$ appear also in $\Sigma^{\prime}$ but with arity 0 . Now, we recursively define $T: \mathcal{T}(\Sigma, \mathcal{X}) \rightarrow \mathcal{T}\left(\Sigma^{\prime}, \mathcal{X}\right)$ as $T(c)=c$ and $T(x)=x$ for constants $c \in \Sigma_{0}$ and variables $x \in \mathcal{X}$, and $T\left(g\left(t_{1}, \ldots, t_{k}\right)\right)=f\left(T\left(t_{1}\right), \ldots, T\left(t_{k}\right), g, \ldots, g\right)$ for terms headed with $g \in \Sigma-\Sigma_{0}$. We denote $\{T(l) \rightarrow T(r) \mid l \rightarrow r \in R\}$ as $T(R)$ for a given TRS $R$. Note that the size of $T(R)$ is at most $m$ times the size of $R$, and hence, this transformation can be easily performed in polynomial time. Note also that $R$ is a TRS over $\Sigma$, and that $T(R)$ is a TRS over $\Sigma^{\prime}$. As we mentioned in the preliminaries, we will not explicitly state the signature of each TRS.

Lemma 3.1. Let $R$ be a TRS. Let $E$ be a permutative theory. Then, $R / E$ is (innermost) terminating if and only if $T(R) / T(E)$ is (innermost) terminating.

Proof. It is straightforward to see that, for any terms $s, t$ of $\mathcal{T}(\Sigma, \mathcal{X}), s \rightarrow_{R} t \Leftrightarrow T(s) \rightarrow_{T(R)}$ $T(t)$ and $s \rightarrow_{E} t \Leftrightarrow T(s) \rightarrow_{T(E)} T(t)$ hold. Thus, non-termination of $R / E$ trivially implies non-termination of $T(R) / T(E)$.

For the left-to-right direction, we define the transformation $T^{\prime}$ as the following extension of the inverse of $T$ on the image. Since $T$ is not surjective, we will use two new function symbols, $\$$ and \# of arity $m$ and 0 respectively, for defining $T^{\prime}(t)$ when $t$ is not in the image of $T$. We define $T^{\prime}: \mathcal{T}\left(\Sigma^{\prime}, \mathcal{X}\right) \rightarrow \mathcal{T}(\Sigma \cup\{\$, \#\}, \mathcal{X})$ as follows:

$$
\begin{aligned}
T^{\prime}(c)=c, T^{\prime}(x)=x & \text { for constants } c \in \Sigma_{0} \text { and variables } x \in \mathcal{X} \\
T^{\prime}(g)=\# & \text { for function symbols } g \in \Sigma-\Sigma_{0} \\
T^{\prime}\left(f\left(t_{1}, \ldots, t_{k}, g, \ldots, g\right)\right)=g\left(T^{\prime}\left(t_{1}\right), \ldots, T^{\prime}\left(t_{k}\right)\right) & \text { for } g \in \Sigma_{k} \\
T^{\prime}\left(f\left(t_{1}, \ldots, t_{m}\right)\right)=\$\left(T^{\prime}\left(t_{1}\right), \ldots, T^{\prime}\left(t_{m}\right)\right) & \text { in all other cases }
\end{aligned}
$$

It is easy to see that any rewrite step $s \rightarrow_{T(R)} t$ can be transformed into a rewrite step $T^{\prime}(s) \rightarrow_{T^{\prime}(T(R))} T^{\prime}(t)$, and any rewrite step $s \rightarrow_{T(E)} t$ can be transformed into a rewrite step $T^{\prime}(s) \rightarrow_{T^{\prime}(T(E))} T^{\prime}(t)$. Thus, non-termination of $T(R) / T(E)$ implies non-termination of $T^{\prime}(T(R)) / T^{\prime}(T(E))$ for the signature $\Sigma \cup\{\$, \#\}$. Note that $T^{\prime}(T(R))$ and $T^{\prime}(T(E))$ are, in fact, $R$ and $E$, respectively. Thus, we conclude that $R / E$ is non-terminating over the signature $\Sigma \cup\{\$, \#\}$. But, note that non-termination (and non-termination modulo) of a TRS does not depend on symbols in the signature that do not occur in the rules. Hence, $R / E$ is non-terminating over the original signature, and we are done.

In the case where $R$ is left-shallow, we will also assume that $R$ is, indeed, left-flat. If this was not the case, we proceed by applying several times the following transformation step a), until $R$ is left-flat. 
step a) If there is a non-constant ground term $u$ that is a proper subterm of a left-hand side of a rule in $R$, then create a new constant $c$, replace all occurrences of $u$ in the left-hand sides of the rules of $R$ by $c$, and add the rule $u \rightarrow c$ to $R$. Formally, the new TRS $R^{\prime}$ is $\{u \rightarrow c\} \cup\left\{\mathrm{NF}_{u \rightarrow c}(l) \rightarrow r \mid(l \rightarrow r) \in R\right\}$. Note that, as a consequence of Assumption (A3), when considering innermost rewriting, $u$ is necessarily a normal form.

We will also assume that all rules in $R$ are right-flat. If this was not the case, as before we proceed by applying several times the following transformation step $b$ ), until the obtained TRS is right-flat.

step b) If there is a non-constant ground term $u$ that is a proper subterm of a right-hand side of a rule in $R$, then create a new constant $c$, replace all occurrences of $u$ in the righthand sides of the rules of $R$ by $c$, and add the rule $c \rightarrow u$ to $R$. Formally, the new TRS $R^{\prime}$ is $\{c \rightarrow u\} \cup\left\{l \rightarrow \mathrm{NF}_{u \rightarrow c}(r) \mid(l \rightarrow r) \in R\right\}$.

Every step (a or b) decreases the total sum of the number of positions at depth more than one in all the left-hand and right-hand sides of $R$. Moreover, also at every step, the total size of the TRS increases by at most the size of two constants. Hence, this process terminates in linear time and the size of the resulting flat TRS is within a constant factor of the size of the original shallow TRS.

Lemma 3.2. Let $R$ be a TRS. Let $E$ be a permutative theory. Let $R^{\prime}$ be obtained from $R$ by applying step a). Then, $R / E$ is (innermost) terminating if and only if $R^{\prime} / E$ is (innermost) terminating.

Proof. For the right-to-left direction, we first observe that each rewrite step $s \rightarrow_{R} t$ can be transformed into a derivation of the form $s \rightarrow_{R^{\prime}}^{+} t$, since the application of a rewrite rule $l \rightarrow r$ can be simulated by several applications of $u \rightarrow c$ and one application of $\mathrm{NF}_{u \rightarrow c}(l) \rightarrow r$. Thus, any derivation of $R \cup E$ with infinitely many rewrite steps of $R$ and starting from a certain term $s$ can be transformed into a derivation of $R^{\prime} \cup E$ with infinitely many rewrite steps of $R^{\prime}$ and starting from $s$. In the case of innermost rewriting, we have that $u$ is a normal form and hence, the transformed derivation is also innermost.

For the left-to-right direction, we first observe the following two facts:

- The existence of a rewrite step $s \rightarrow_{u \rightarrow c} t$ implies $\mathrm{NF}_{c \rightarrow u}(s)=\mathrm{NF}_{c \rightarrow u}(t)$.

- For each rule $l \rightarrow r$ of $R$, if $l^{\prime}=\mathrm{NF}_{u \rightarrow c}(l)$, then, for each rewrite step $s \rightarrow_{l^{\prime} \rightarrow r} t$, it holds that $\mathrm{NF}_{c \rightarrow u}(s) \rightarrow_{l \rightarrow r} \mathrm{NF}_{c \rightarrow u}(t)$.

From the above facts, it follows that any rewrite step $s \rightarrow_{R^{\prime}} t$ can be transformed into a derivation $\mathrm{NF}_{c \rightarrow u}(s) \rightarrow{ }_{R}^{*} \mathrm{NF}_{c \rightarrow u}(t)$ with 0 or 1 steps. Note that this is not enough to argue that a derivation of $R^{\prime} \cup E$ with infinitely many steps with $R^{\prime}$, and starting from a term $s$, can be transformed into a derivation of $R \cup E$ with infinitely many steps with $R$ starting from $\mathrm{NF}_{c \rightarrow u}(s)$. This is because rewrite steps with $u \rightarrow c$ are, in fact, removed. However, it suffices to additionally argue that a derivation of $\{u \rightarrow c\} \cup E$ with infinitely many steps of $\{u \rightarrow c\}$ cannot exist. This is a consequence of the fact that rules of $E$ preserve the size, and $u \rightarrow c$ decreases the size. Finally, in case of innermost rewriting, using the facts that $u$ is a normal form, $s \rightarrow_{R^{\prime}} t$ is an innermost step, and $l^{\prime}=\mathrm{NF}_{u \rightarrow c}(l)$, we infer that the transformed derivation is an innermost derivation.

The preservation of termination for the case of step b) is proved analogously. 
Lemma 3.3. Let $R$ be a TRS. Let $E$ be a permutative theory. Let $R^{\prime}$ be obtained from $R$ by applying step $b$ ). Then, $R / E$ is (innermost) terminating if and only if $R^{\prime} / E$ is (innermost) terminating.

\section{Right Flat TRS}

In this section, we will show that, given a right-flat TRS $R$ and a term $s$, it is decidable if $R$ is terminating from $s$. In particular, this implies that non-termination is semi-decidable for right-flat TRS. We will show that termination is undecidable for right-flat TRS in Section 8 ,

The proofs of this section are written for general termination, but they are also valid when we interpret termination as innermost termination, reachability as innermost reachability, and so on.

An important property of a right-flat TRS $R$ is that whenever $s \rightarrow_{R}^{*} t$ holds, then every subterm of $t$ is reachable from either a constant or some subterm of $s$. This result, stated as Lemma 4.4, is used extensively later. It is proved by inductively marking each position of a term (in the above derivation) by a term from $\operatorname{Subterms}(s) \cup \Sigma_{0}$. The idea of the marking $t_{p}$ at each position $p$ of $t$ is that it satisfies $\left.t_{p} \rightarrow_{R}^{*} t\right|_{p}$, and moreover, for a position $p^{\prime}>p$ the corresponding marking $t_{p^{\prime}}$ of $t$ at $p^{\prime}$ is context-reachable from $t_{p}$.

Definition 4.1. Let $R$ be a right-flat TRS. Let $s=s_{1} \rightarrow_{R} \ldots \rightarrow_{R} s_{n}$ be a derivation with $R$. A Marking of this derivation is a sequence $M_{1}, \ldots, M_{n}$ of $n$ functions $M_{i}: \operatorname{Pos}\left(s_{i}\right) \mapsto$ Subterms $(s) \cup \Sigma_{0}$ defined inductively as follows:

- For each $p$ in $\operatorname{Pos}(s)$, we define $M_{1}(p)=\left.s\right|_{p}$.

- For $1 \leq i<n$ we assume that $M_{i}$ is defined. Let $s_{i} \rightarrow_{l \rightarrow r, \bar{p}} s_{i+1}$ be the $i$ th rewrite step of the derivation above more explicitly written. Then, we define $M_{i+1}$ as follows:

(i) For each $p$ in $\operatorname{Pos}\left(s_{i+1}\right)$ satisfying $p \ngtr \bar{p}$, we define $M_{i+1}(p):=M_{i}(p)$.

(ii) For each $p$ in $\operatorname{Pos}\left(s_{i+1}\right)$ satisfying $p=\bar{p} \cdot p_{0},\left|p_{0}\right|=1$, and $\left.r\right|_{p_{0}}$ is a constant, we define $M_{i+1}(p):=\left.r\right|_{p_{0}}$.

(iii) For each $p$ in $\operatorname{Pos}\left(s_{i+1}\right)$ satisfying $p=\bar{p} \cdot p_{0} \cdot p_{1},\left|p_{0}\right|=1,\left|p_{1}\right| \geq 0$, and $\left.r\right|_{p_{0}}$ is a variable, we define $M_{i+1}(p):=M_{i}\left(\bar{p} \cdot q_{0} \cdot p_{1}\right)$, where $q_{0}$ is any position in $l$ such that $\left.l\right|_{q_{0}}=\left.r\right|_{p_{0}}$.

Recall that we are assuming that every variable on the right-hand side also appears on the left-hand side; if not, then the TRS is trivially non-terminating. The following example illustrates the definition of marking and also shows that markings need not be unique.

Example 4.2 (Marking). Let $R:=\{a \rightarrow f(c), b \rightarrow f(c), g(x, x) \rightarrow f(x)\}$ and consider the derivation $s_{1}:=g(a, b) \rightarrow s_{2}:=g(f(c), b) \rightarrow s_{3}:=g(f(c), f(c)) \rightarrow s_{4}:=f(f(c))$. A marking for this derivation is given by: $M_{1}(p)=\left.s_{1}\right|_{p}$ for all $p \in \operatorname{Pos}\left(s_{1}\right), M_{2}(p)=M_{1}(p)$ for all $p \in \operatorname{Pos}\left(s_{2}\right)-\{11\}, M_{2}(11)=c, M_{3}(p)=M_{2}(p)$ for all $p \in \operatorname{Pos}\left(s_{3}\right)-\{21\}$, $M_{3}(21)=c, M_{4}(\lambda)=s_{1}, M_{4}(1)=a$, and $M_{4}(11)=c$. Note that if we redefined $M_{4}(1)$ so that $M_{4}(1)=b$, then the resulting functions would still be a marking. Hence, there can be multiple markings for the same derivation.

Now we will state and prove some useful properties about markings. Henceforth, let us fix $R$ to be a right-flat TRS, $s=s_{1} \rightarrow_{R} \ldots \rightarrow_{R} s_{n}$ to be a (innermost) derivation and $M_{1}, \ldots, M_{n}$ to be a marking of this derivation. The properties below will capture the intuition that, if $M_{i}(p)=t$, then the term $\left.s_{i}\right|_{p}$ is reachable from the term $t$. 
Lemma 4.3. $M_{n}(\lambda)=s$. Moreover, if $s$ is not a constant, then, for each $p$ in $\operatorname{Pos}\left(s_{n}\right)-\{\lambda\}$ we have $M_{n}(p) \neq s$.

Proof. The claim is proved by induction on $n$. For $n=1$, by definition of marking, we have $M_{1}(\lambda)=\left.s_{1}\right|_{\lambda}=s$. Moreover, if $s$ is not a constant, for each $p$ in $\operatorname{Pos}\left(s_{1}\right)-\{\lambda\}$ we have $M_{1}(p)=\left.s_{1}\right|_{p}=\left.s\right|_{p} \neq s$.

For the induction step, suppose $s_{n} \rightarrow_{l \rightarrow r, \bar{p}} s_{n+1}$. By induction hypothesis, we know that $M_{n}(\lambda)=s$ and whenever $s$ is not a constant then, for each $p \in \operatorname{Pos}\left(s_{n}\right)-\{\lambda\}$, $M_{n}(p) \neq s$ holds. The fact that $M_{n+1}(\lambda)=s$ follows from the fact that $M_{n}(\lambda)=s$ and $M_{n+1}(\lambda)=M_{n}(\lambda)$ holds, since Case (i) of the definition of marking applies for $p=\lambda$. Under the assumption that $s$ is not a constant, we note that Case (ii) defines $M_{n+1}(p)$ as a constant, and cases (i) and (iii) define $M_{n+1}(p)$ for $p$ in $\operatorname{Pos}\left(s_{n+1}\right)-\{\lambda\}$ as $M_{n}\left(p^{\prime}\right)$ for some $p^{\prime}$ in $\operatorname{Pos}\left(s_{n}\right)-\{\lambda\}$. Thus, from the assumption that $M_{n}\left(p^{\prime}\right) \neq s$, it follows that $M_{n+1}(p) \neq s$.

A second property of markings is that $\left.s_{n}\right|_{p}$ is always reachable from $M_{n}(p)$.

Lemma 4.4. For each $p$ in $\operatorname{Pos}\left(s_{n}\right),\left.s_{n}\right|_{p}$ is (innermost) reachable from $M_{n}(p)$.

Proof. The claim is proved by induction on $n$. For $n=1$, by definition of marking, we have $M_{1}(p)=\left.s_{1}\right|_{p}$ for each $p$ in $\operatorname{Pos}\left(s_{1}\right)$. Thus, $\left.M_{1}(p) \rightarrow_{R}^{*} s_{1}\right|_{p}$ in 0 steps follows trivially.

For the induction step, suppose $s_{n} \rightarrow_{l \rightarrow r, \bar{p}} s_{n+1}$ is the $n$-th (innermost) rewrite step. By induction hypothesis, $\left.M_{n}(p) \rightarrow_{R}^{*} s_{n}\right|_{p}$ holds for each $p \in \operatorname{Pos}\left(s_{n}\right)$. Consider a fixed $p \in \operatorname{Pos}\left(s_{n+1}\right)$. We prove $\left.M_{n+1}(p) \rightarrow_{R}^{*} s_{n+1}\right|_{p}$ as follows:

- If $p \leq \bar{p}$, then we have $M_{n+1}(p)=\left.\left.M_{n}(p) \rightarrow_{R}^{*} s_{n}\right|_{p} \rightarrow_{l \rightarrow r, \bar{p}-p} s_{n+1}\right|_{p}$. Note that, since $s_{n}$ (innermost) rewrites to $s_{n+1}$, it follows that $\left.s_{n}\right|_{p}$ (innermost) rewrites to $\left.s_{n+1}\right|_{p}$.

- If $p=\bar{p} \cdot p_{0}$ and $\left|p_{0}\right|=1$ hold, and $\left.r\right|_{p_{0}}$ is a constant, then, by definition of marking we have $M_{n+1}(p)$ is $\left.r\right|_{p_{0}}$, from which $\left.M_{n+1}(p) \rightarrow_{R}^{*} r\right|_{p_{0}}=\left.s_{n+1}\right|_{p}$ in 0 steps follows trivially.

- If $p=\bar{p} \cdot p_{0} \cdot p_{1}$ and $\left|p_{0} \cdot p_{1}\right| \geq 1$ hold, and $\left.r\right|_{p_{0}}$ is a variable, then, for some $q_{0}, M_{n+1}(p)=$ $M_{n}\left(\bar{p} \cdot q_{0} \cdot p_{1}\right)$ and $\left.s_{n+1}\right|_{p}=\left.s_{n}\right|_{\bar{p} \cdot q_{0} \cdot p_{1}}$ hold, and $\left.M_{n}\left(\bar{p} \cdot q_{0} \cdot p_{1}\right) \rightarrow_{R}^{*} s_{n}\right|_{\bar{p} \cdot q_{0} \cdot p_{1}}$ holds by induction hypothesis. Thus, $\left.M_{n+1}(p) \rightarrow_{R}^{*} s_{n+1}\right|_{p}$ follows.

- If $p \| \bar{p}$, then the claim holds by induction hypothesis again as $\left.s_{n+1}\right|_{p}=\left.s_{n}\right|_{p}$ and $M_{n+1}(p)=M_{n}(p)$ hold.

Thus, for each position $p \in \operatorname{Pos}\left(s_{n+1}\right)$, we proved that $\left.s_{n+1}\right|_{p}$ is (innermost) reachable from $M_{n+1}(p)$.

Corollary 4.5. If $s$ is a constant, then all subterms of $s_{n}$ are (innermost) reachable from a constant.

Another property of markings is that $M_{n}(p)$ is context-reachable from $M_{n}\left(p^{\prime}\right)$ for all $p^{\prime} \leq p$.

Lemma 4.6. For each $p, p^{\prime} \in \operatorname{Pos}\left(s_{n}\right)$ satisfying $p^{\prime}<p, M_{n}(p)$ is (innermost) contextreachable from $M_{n}\left(p^{\prime}\right)$. Moreover, if $M_{n}(p)$ and $M_{n}\left(p^{\prime}\right)$ are both constants, then $M_{n}(p)$ is (innermost) context-reachable from $M_{n}\left(p^{\prime}\right)$ with a non-empty context.

Proof. The claim is proved by induction on $n$. For $n=1$, by definition of marking we have $M_{1}(p)=\left.s_{1}\right|_{p}$ for each $p$ in $\operatorname{Pos}\left(s_{1}\right)$. Since, for each $p, p^{\prime} \in \operatorname{Pos}\left(s_{1}\right)$ satisfying $p^{\prime}<p$, $\left.s_{1}\right|_{p^{\prime}}=\left.s_{1}\right|_{p^{\prime}}\left[\left.s_{1}\right|_{p}\right]_{p-p^{\prime}}$ holds, then we also have $M_{1}\left(p^{\prime}\right)=M_{1}\left(p^{\prime}\right)\left[M_{1}(p)\right]_{p-p^{\prime}}$. Thus, the statement trivially follows for the base case. 
For the induction step, suppose $s_{n} \rightarrow_{l \rightarrow r, \bar{p}} s_{n+1}$ is the $n$ 'th (innermost) rewrite step. Consider two fixed positions $p, p^{\prime} \in \operatorname{Pos}\left(s_{n+1}\right)$ satisfying $p^{\prime}<p$. We distinguish the following cases.

- If $p \leq \bar{p}$ or $p \| \bar{p}$, then we have $M_{n+1}(p)=M_{n}(p)$ and $M_{n+1}\left(p^{\prime}\right)=M_{n}\left(p^{\prime}\right)$. Thus, the statement follows by induction hypothesis.

- If $p=\bar{p} \cdot p_{0},\left|p_{0}\right|=1$, and $\left.r\right|_{p_{0}}$ is a constant, then $M_{n+1}(p)=\left.r\right|_{p_{0}}$ holds. By Lemma 4.4, $\left.s_{n+1}\right|_{p^{\prime}}$ is reachable from $M_{n+1}\left(p^{\prime}\right)$. Note that $M_{n+1}(p)$ is a proper subterm of $\left.s_{n+1}\right|_{p^{\prime}}$. Hence, $M_{n+1}(p)$ is context reachable from $M_{n+1}\left(p^{\prime}\right)$ with a non-empty context (independently of whether $M_{n+1}\left(p^{\prime}\right)$ is a constant or not).

- If $p=\bar{p} \cdot p_{0} \cdot p_{1},\left|p_{0} \cdot p_{1}\right| \geq 1$ and $\left.r\right|_{p_{0}}$ is a variable, then, for some $q_{0}, M_{n+1}(p)=M_{n}\left(\bar{p} \cdot q_{0} \cdot p_{1}\right)$. We distinguish two cases. (a) If $p^{\prime} \leq \bar{p}$, then $M_{n+1}\left(p^{\prime}\right)=M_{n}\left(p^{\prime}\right)$ and, by induction hypothesis, $M_{n}\left(\bar{p} \cdot q_{0} \cdot p_{1}\right)$ is context reachable from $M_{n}\left(p^{\prime}\right)$ (with a non-empty context if both $M_{n}\left(p^{\prime}\right)$ and $M_{n}\left(\bar{p} \cdot q_{0} \cdot p_{1}\right)$ are constants), which is the same as saying that $M_{n+1}(p)$ is context reachable from $M_{n+1}\left(p^{\prime}\right)$ (with a non-empty context if both $M_{n+1}\left(p^{\prime}\right)$ and $M_{n+1}(p)$ are constants). (b) If $p^{\prime}>\bar{p}$ holds, then $M_{n+1}\left(p^{\prime}\right)=M_{n}\left(\bar{p} \cdot q_{0} \cdot p_{1}^{\prime}\right)$ holds for some $p_{1}^{\prime}<p_{1}$ and, by induction hypothesis, $M_{n}\left(\bar{p} \cdot q_{0} \cdot p_{1}\right)$ is context reachable from $M_{n}\left(\bar{p} \cdot q_{0} \cdot p_{1}^{\prime}\right)$ (with a non-empty context if both $M_{n}\left(\bar{p} \cdot q_{0} \cdot p_{1}^{\prime}\right)$ and $M_{n}\left(\bar{p} \cdot q_{0} \cdot p_{1}\right)$ are constants). This is the same as saying that $M_{n+1}(p)$ is context reachable from $M_{n+1}\left(p^{\prime}\right)$ (with a non-empty context if both $M_{n+1}\left(p^{\prime}\right)$ and $M_{n+1}(p)$ are constants).

Thus, in all cases, the claim follows.

We illustrate Lemma 4.6 by an example below.

Example 4.7 (Lemma 4.6). Consider again the derivation and marking defined in Example 4.2. We note that, on the term $s_{4}:=f(f(c))$, we had the marking $M_{4}$ defined so that $M_{4}(1)=a$ and $M_{4}(11)=c$. By Lemma 4.6, $c$ should be context-reachable from $a$, and indeed we have $a \rightarrow f(c)$.

Finally, another observation about a marking is that positions below height $(s)$ are always marked by constants.

Lemma 4.8. For each $p \in \operatorname{Pos}\left(s_{n}\right)$ such that $|p|>\operatorname{height}(s), M_{n}(p)$ is a constant.

Proof. The claim is proved by induction on $n$. For $n=1$, note that $s_{1}=s$ holds and hence all $p \in \operatorname{Pos}\left(s_{1}\right)$ satisfy $|p| \leq$ height $(s)$. Thus, the claim is vacuously true.

For the induction step, suppose $s_{n} \rightarrow_{l \rightarrow r, \bar{p}} s_{n+1}$ is the $n$ 'th (innermost) rewrite step. Consider any position $p \in \operatorname{Pos}\left(s_{n+1}\right)$ satisfying $|p|>$ height $(s)$.

- If $p \| \bar{p}$ or $p \leq \bar{p}$, then $M_{n+1}(p)=M_{n}(p)$ holds, and by induction hypothesis $M_{n}(p)$ is a constant.

- If $p=\bar{p} \cdot p_{0},\left|p_{0}\right|=1$ and $\left.r\right|_{p_{0}}$ is a constant, then $M_{n+1}(p)=\left.r\right|_{p_{0}}$ holds, which is a constant.

- If $p=\bar{p} \cdot p_{0} \cdot p_{1},\left|p_{0} \cdot p_{1}\right| \geq 1$ and $\left.r\right|_{p_{0}}$ is a variable, then $M_{n+1}(p)=M_{n}\left(\bar{p} \cdot q_{0} \cdot p_{1}\right)$ for some $q_{0}$, and $\left|\bar{p} \cdot q_{0} \cdot p_{1}\right| \geq|p|$ holds since left-hand sides of $R$ are not variables and $R$ is right-flat. Hence, the induction hypothesis is applicable and we can conclude that $M_{n}\left(\bar{p} \cdot q_{0} \cdot p_{1}\right)$, and therefore $M_{n+1}(p)$, is a constant.

Thus, for all $p$ s.t. $|p|>$ height $(s), M_{n+1}(p)$ is a constant. This completes the proof.

An important consequence of Lemma 4.6 and Lemma 4.8 is that, if $R$ is terminating from $s$, then the height of terms reachable from $s$ is bounded by the height of $s$ plus the number of constants in $\Sigma$. 
Corollary 4.9. Let $R$ be a right-flat TRS. Let $E$ be a permutative theory. Let $s$ be a term such that $R / E$ is (innermost) terminating from $s$. Then for any term $t$ (innermost) reachable from $s$ with $R / E$, we have height $(t) \leq$ height $(s)+\left|\Sigma_{0}\right|$.

Proof. We proceed by contradiction by assuming $s \rightarrow_{R / E}^{*} t$ and height $(t)>\operatorname{height}(s)+$ $\left|\Sigma_{0}\right|$. Recall that the derivation $s \rightarrow_{R / E}^{*} t$ can be seen as a derivation $s \rightarrow_{R \cup E}^{*} t$. Let $M_{1}, \ldots, M_{n}$ be a marking of this derivation $s \rightarrow_{R \cup E}^{*} t$. By Lemma4.8, each position in $t$ that is deeper than height $(s)$ is marked with a constant. Since height $(t)>\operatorname{height}(s)+\left|\Sigma_{0}\right|$ holds, by pigeon-hole principle, there are two positions $p, p^{\prime} \in \operatorname{Pos}(t)$ such that $p<p^{\prime}$ and $M_{n}(p)=M_{n}\left(p^{\prime}\right)$ hold, and $M_{n}(p)$ is a constant, say $c$. By Lemma 4.6, it follows that $c$ is context reachable from $c$ with a non-empty context. Moreover, since $E$ is a permutative theory, $c$ is context reachable from $c$ with a derivation using at least one rewrite step with a rule of $R$. Furthermore, by Lemma 4.3 the position $\lambda$ of every term in a derivation is marked with $s$. Using Lemma 4.6 again, we infer that $M_{n}(p)=c$, is also context reachable from $s$. Thus, we can construct a derivation $s \rightarrow_{R \cup E}^{*} C_{1}[c] \rightarrow{ }_{R \cup E}^{+}$ $C_{1}\left[C_{2}[c]\right] \rightarrow_{R \cup E}^{+} C_{1}\left[C_{2}\left[C_{2}[c]\right]\right] \rightarrow_{R \cup E}^{+} \cdots$ with infinitely many steps with $R$. Hence, there is a derivation starting from $s$ using $R / E$ with infinitely many rewrite steps, a contradiction.

Using the above corollary, we can show that the existence of non-terminating derivations starting from a term is decidable for right-flat TRS.

Theorem 4.10. Termination (innermost termination) of a right-flat TRS $R$ modulo a permutative theory $E$ from a given term is decidable. Hence, non-termination (innermost non-termination) is semi-decidable for right-flat TRS modulo permutative theories.

Proof. Let $s$ be any term. We enumerate all (innermost) derivations starting from $s$. If we reach a term with height greater than height $(s)+\left|\Sigma_{0}\right|$, then by Corollary 4.9 we know that $R / E$ is non-terminating from $s$. Otherwise, we will get only finitely many reachable terms. If there is a derivation that cycles among these terms, then $R / E$ is non-terminating from $s$. If not, then $R / E$ is terminating from $s$.

Remark: We can use an argument similar to the one used in the proof of Theorem 4.10 to prove that, for any class $\mathcal{C}$ of TRS's that are effectively regularity preserving, termination of a TRS $R$ of $\mathcal{C}$ from a term $s$, where both $R$ and $s$ are given as input, is decidable. While we do not use this observation here, we nevertheless note that, using recent results on regularity preserving TRSs [TKS00, we immediately get very simple proofs of known decidability results, such as for right-ground TRS [Der81: a right-ground TRS is regularity preserving, and is non-terminating iff it is non-terminating from some right-hand side, which can be checked for every right-hand side term using the above observation.

\section{InNeRmost TERmination of Flat TRS's}

In this section, we show that innermost termination of flat TRS modulo permutative theories is decidable. In sharp contrast, general termination is undecidable for flat TRS (Section 8).

Let $R$ be a flat TRS, and let $E$ be a permutative theory. We show decidability of innermost termination of $R / E$ by showing that if $R / E$ is not innermost terminating, then there will be an infinite $R / E$ derivation starting from a ground flat term. Using Theorem 4.10 , we know that this latter check is decidable. 
Lemma 5.1. Let $R$ be a flat TRS. Let $E$ be a permutative theory. Suppose that $R / E$ is not innermost terminating. Then, there is an innermost derivation starting from a ground flat term with infinitely many innermost rewrite steps.

Proof. We assume that there is no innermost derivation with infinitely many innermost rewrite steps and starting from a constant, and we show that there is one from a ground flat term with height 1 .

Since $R / E$ is not innermost terminating, there exists an innermost derivation $t_{0} \rightarrow R \cup E$ $t_{1} \rightarrow R \cup E \ldots$ with infinitely many innermost rewrite steps using $R$, whose first step is at position $\lambda$. We first prove that for every $i$, every subterm at depth 1 of $t_{i}$ is either reachable from a constant, or a normal form. First note that no term $t_{i}$ is a constant, by our initial assumption. Moreover, since we use innermost rewriting, all proper subterms of $t_{0}$ are normal forms. By Lemma 4.4, all subterms at depth 1 of $t_{i}$ are innermost reachable from either constants or proper subterms of $t_{0}$. Hence they are innermost reachable from constants, or they are normal forms.

Now, we note that there exists at least one constant, call it $c$, that is a normal form. If not, any ground term can be innermost rewritten to another ground term, and hence there will be innermost derivations starting from constants with infinitely many innermost rewrite steps, which contradicts our initial assumption.

We construct a new innermost derivation $t_{0}^{\prime} \rightarrow_{R \cup E, \lambda} t_{1}^{\prime} \rightarrow_{R \cup E} \ldots$ by defining each $t_{i}^{\prime}$ to be as $t_{i}$ but replacing every subterm at depth 1 that is not innermost reachable from any constant by the constant $c$ chosen above. We need to show that the new derivation is "correct", that is, there is an innermost rewrite step from $t_{i-1}^{\prime}$ to $t_{i}^{\prime}$. Consider the corresponding innermost rewrite step $t_{i-1} \rightarrow_{l \rightarrow r, \bar{p}} t_{i}$.

- If $\bar{p}$ is not $\lambda$, then $\bar{p}$ is of the form $j$.p for some $j$ in $\{1, \ldots, m\}$ and some position $p$. Since $\left.t_{i-1}\right|_{j}$ is rewritten, it is not a normal form. Thus it is innermost reachable from a constant, and hence, $\left.t_{i-1}^{\prime}\right|_{j}$ and $\left.t_{i}^{\prime}\right|_{j}$ coincide with $\left.t_{i-1}\right|_{j}$ and $\left.t_{i}\right|_{j}$, respectively. Therefore, the same innermost rewrite step can be applied on $t_{i-1}^{\prime}$ to produce $t_{i}^{\prime}$.

- If $\bar{p}$ is $\lambda$, then, by our initial assumption, both $l$ and $r$ are not constants. Moreover, $r$ cannot be a variable, since, otherwise, $t_{i}$ would be a normal form since we have innermost rewriting (and the derivation would be finite). Hence, $l \rightarrow r$ is of the form $f\left(\alpha_{1}, \ldots, \alpha_{m}\right) \rightarrow f\left(\beta_{1}, \ldots, \beta_{m}\right)$. Recall that, since $R$ is flat, each $\alpha_{i}$ and each $\beta_{i}$ is either a constant or a variable. If $\sigma$ is the substitution used in this innermost rewrite step, then define $\sigma^{\prime}$ to be as $\sigma$ except for the cases where $\sigma(x)$ is not innermost reachable from a constant, in which case we define $\sigma^{\prime}(x)=c$. With these definitions, $t_{i-1}^{\prime} \rightarrow_{l \rightarrow r, \sigma^{\prime}, \lambda} t_{i}^{\prime}$ is an innermost rewrite step.

The derivation $t_{0}^{\prime} \rightarrow_{R \cup E} t_{1}^{\prime} \rightarrow_{R \cup E} \ldots$ is again innermost, has infinitely many innermost rewrite steps with $R$, and the initial term $t_{0}^{\prime}$ satisfies that all its subterms at depth 1 are innermost reachable from constants. Therefore, there exists a ground flat term $s$ with height 1 such that $s \rightarrow_{R \cup E}^{*} t_{0}^{\prime}$ is an innermost derivation, and hence, there exists an innermost derivation with infinitely many innermost rewrite steps starting from a ground flat term $s$ with height 1.

Theorem 5.2. Innermost termination modulo permutative theories is decidable for shallow TRS's.

Proof. By Lemmas 3.1, 3.2 and 3.3 innermost termination of shallow TRS modulo permutative theories can be reduced to the particular case where $R$ is flat and where the signature contains just one non-constant function symbol. 
Since there are only finitely many ground flat terms, using Theorem 4.10, we can check if a given flat $R / E$ is not innermost terminating starting from one of these terms. By Lemma 5.1, we will find a witness for non-termination this way iff $R / E$ is not innermost terminating.

\section{Termination and Innermost Termination of Right-Flat Right-Linear TRS'S}

In this section, we show decidability of termination and innermost termination for right-flat right-linear TRS. Again, the proofs of this section are written for general rewriting, but they remain valid for innermost rewriting.

The proof of decidability of (innermost) termination for right-flat right-linear TRS depends on two key observations. The first one is Lemma 4.4, which says that for any (innermost) derivation $s \rightarrow_{R}^{*} t$ using a right-flat TRS $R$, every proper subterm of $t$ is (innermost) reachable from either a constant or a proper subterm of $s$. The second key lemma is stated by first defining the following measure of a term $t$ :

$$
\|t\|:=\mid\left\{p|p \in \operatorname{Pos}(t) \wedge t|_{p} \text { is not (innermost) reachable from a constant }\right\} \mid
$$

Note that $\|t\|$ depends on whether we are dealing with general or innermost rewriting.

Let us fix $R$ to be a right-flat right-linear TRS and $E$ to be a permutative theory. The first lemma below uses right-linearity of $R$.

Lemma 6.1. If $s \rightarrow_{R} t$, then $\|s\| \geq\|t\|$. Moreover, if $s\left[f\left(s_{1}, \ldots, s_{m}\right)\right]_{\bar{p}}$ rewrites to $t$ at position $\bar{p}$ with a rule $f\left(l_{1}, \ldots, l_{m}\right) \rightarrow r$, and $\|s\|=\|t\|$, then, for every $i$ in $\{1 \ldots m\}$, if $s_{i}$ is not reachable from a constant, then $l_{i}$ is a variable.

Proof. Let $s \rightarrow_{l \rightarrow r, \bar{p}} t$ be the rewrite step of the lemma. We prove the first statement by constructing an injective map, from positions $p$ of $\operatorname{Pos}(t)$ such that $\left.t\right|_{p}$ is not reachable from a constant, to positions $p^{\prime}$ of $s$ such that $\left.s\right|_{p^{\prime}}$ is not reachable from a constant, as follows. If $p \| \bar{p}$ or $p \leq \bar{p}$, then we let $p^{\prime}:=p$. If $p>\bar{p}$, then $p$ can be written in the form $\bar{p} \cdot p_{0} \cdot p_{1}$ where $\left.r\right|_{p_{0}}$ is a height 0 term. In fact, $\left.r\right|_{p_{0}}$ cannot be a constant since otherwise $\left.t\right|_{p}$ would be a constant. Hence, $\left.r\right|_{p_{0}}$ is a variable. We choose a position $p_{0}^{\prime}$ such that $\left.l\right|_{p_{0}^{\prime}}$ is the same variable as $\left.r\right|_{p_{0}}$ and define $p^{\prime}:=\bar{p} \cdot p_{0}^{\prime} \cdot p_{1}$. The injectivity of the map follows by right-linearity of $R$. Hence, $\|s\| \geq\|t\|$ holds.

For the second statement, we assume $\|s\|=\|t\|$, that $s$ is of the form $s\left[f\left(s_{1}, \ldots, s_{m}\right)\right]_{\bar{p}}$, and $l$ is of the form $f\left(l_{1}, \ldots, l_{m}\right)$. If a certain $s_{i}$ is not reachable from a constant, but $l_{i}$ is not a variable, then $\bar{p} . i$ is not in the image of the previous mapping, and hence $\|s\|>\|t\|$ holds, contradicting $\|s\|=\|t\|$. Therefore, all such $l_{i}$ 's are variables.

Note that since $E$ is linear and flat, Lemma 6.1 applies to rewrite steps with $E$ too. In the next lemma, if $R / E$ is non-terminating, we establish the existence of a non-terminating derivation with certain properties.

Lemma 6.2. If $R / E$ is (innermost) non-terminating and there is no (innermost) nonterminating derivation starting from a constant, then there is an infinite derivation $t_{0} \rightarrow_{R \cup E}$ $t_{1} \rightarrow_{R \cup E} \ldots$ with infinitely many rewrites with $R$ and with the following properties:

(a) there is no infinite derivation starting from a proper subterm of $t_{0}$

(b) there is no rewrite with a collapsing rule at position $\lambda$

(c) there are infinitely many rewrites at position $\lambda$ 
Proof. Since $R / E$ is non-terminating, there exists a derivation $t_{0} \rightarrow_{R \cup E} t_{1} \rightarrow_{R \cup E} \ldots$ with infinitely many rewrite steps with $R$. We pick the derivation that has minimal height for $t_{0}$. We claim this derivation has all the properties mentioned above.

It has Property (a) due to our choice of the infinite derivation. Next assume that $t_{i-1} \rightarrow_{R} t_{i}$ is the first application of a collapsing rule at $\lambda$ in the derivatin. Then, by Lemma 4.3 and Lemma 4.4 all proper subterms of $t_{i-1}$ are reachable from either a constant or a proper subterm of $t_{0}$. Since $t_{i}$ is a proper subterm of $t_{i-1}$, it is reachable from either a constant or a proper subterm of $t_{0}$. In either case we infer the existence of a derivation starting from a term with height smaller than $t_{0}$, and involving infinitely many rewrite steps with $R$, which contradicts our choice of $t_{0}$. Hence, the infinite derivation we picked has Property (b).

Finally, we show that there are infinitely many rewrite steps at position $\lambda$. Suppose not. Let $t_{i-1} \rightarrow_{R \cup E} t_{i}$ be the last rewrite step at position $\lambda$. Then, there is a derivation starting from some subterm at depth 1 of $t_{i}$ with infinitely many rewrite steps with $R$. As before, this subterm is reachable from either a constant or a proper subterm of $t_{0}$. Again, this implies the existence of an infinite derivation that starts from a term with height smaller than $t_{0}$. This contradicts the minimality of $t_{0}$.

The idea of the decidability proof is the same as that for Theorem 5.2 , that is, we show that if $R / E$ is non-terminating, then it is non-terminating from a ground flat term.

Lemma 6.3. If $R / E$ is non-terminating (innermost non-terminating), then there exists an (innermost) derivation starting from a ground flat term with infinitely many rewrite steps.

Proof. Assume that there is no infinite derivation starting from a constant. We will show that there is one from a ground flat term.

Using Lemma 6.2, we know there is an infinite derivation, $t_{0} \rightarrow_{R \cup E} t_{1} \rightarrow_{R \cup E} \ldots$, with Properties (a), (b) and (c) from Lemma 6.2. All the infinitely many rewrite steps at position $\lambda$ in this derivation necessarily are done using rules of the form $l \rightarrow f\left(\alpha_{1}, \ldots, \alpha_{m}\right)$, where the height of $l$ is greater than or equal to 1 . (If not, then there will be a constant in the derivation.) By Lemma 6.1, $\left\|t_{i-1}\right\| \geq\left\|t_{i}\right\|$ for all $i$. Since this relation can not be indefinitely decreasing, for some $n$ we have $\left\|t_{n}\right\|=\left\|t_{n+1}\right\|=\left\|t_{n+2}\right\|=\ldots$. From the derivation $t_{n} \rightarrow_{R \cup E} t_{n+1} \rightarrow_{R \cup E} \ldots$ we construct a new derivation $t_{n}^{\prime} \rightarrow_{R \cup E}^{0,1} t_{n+1}^{\prime} \rightarrow_{R \cup E}^{0,1} \ldots$ with also infinitely many rewrite steps as follows. Analogously to the proof of Lemma 5.1 , we can deduce the existence of at least one constant $c$ that is a normal form. For every $t_{i}$, we construct $t_{i}^{\prime}$ to be equal to $t_{i}$ except for the subterms at depth 1 that are not reachable from constants, which are replaced by $c$. Formally, $t_{i}^{\prime}=t_{i}[c]_{j_{1}} \ldots[c]_{j_{k}}$ if $\left.t_{i}\right|_{j_{1}}, \ldots,\left.t_{i}\right|_{j_{k}}$ are the subterms at depth 1 in $t_{i}$ that are not reachable from constants.

We show that the new derivation is correct by analyzing each rewrite step $t_{i-1} \rightarrow_{R \cup E} t_{i}$ and its corresponding step $t_{i-1}^{\prime} \rightarrow{ }_{R \cup E}^{0,1} t_{i}^{\prime}$.

(1) If $t_{i-1} \rightarrow_{R \cup E} t_{i}$ is done at a position inside a subterm at depth 1 of $t_{i-1}$ that is reachable from a constant, then, the same rewrite step can be applied on $t_{i-1}^{\prime}$ to produce $t_{i}^{\prime}$.

(2) If $t_{i-1} \rightarrow R \cup E t_{i}$ is done at a position inside a subterm, say $\left.t_{i-1}\right|_{j}$, at depth 1 of $t_{i-1}$ that is not reachable from a constant, then, $\left.t_{i}\right|_{j}$ is neither reachable from a constant. This follows from $\left\|\left.t_{i}\right|_{j}\right\|=\left\|\left.t_{i-1}\right|_{j}\right\| \geq 1$ and the fact that, by Lemma 4.5, if a term is reachable from a constant, then all its subterms are. Thus, $t_{i-1}^{\prime}=t_{i}^{\prime}$ holds, and hence, $t_{i-1}^{\prime} \rightarrow^{0} t_{i}^{\prime}$ holds. 
(3) If $t_{i-1} \rightarrow_{R \cup E} t_{i}$ is done at position $\lambda$, then, by Lemma 6.1, if $f\left(l_{1}, \ldots, l_{m}\right) \rightarrow r$ and $\sigma$ are the rule and substitution applied, then $l_{k}$ is a variable for every position $k$ such that $\left.t_{i-1}\right|_{k}$ is not reachable from a constant. We define a new substitution $\sigma^{\prime}$ to be equal to $\sigma$ except for such variables $l_{k}$, for which we define $\sigma^{\prime}\left(l_{k}\right)=c$. The same rule $f\left(l_{1}, \ldots, l_{m}\right) \rightarrow r$ applied to $t_{i-1}^{\prime}$ at position $\lambda$ and with substitution $\sigma^{\prime}$ produces $t_{i}^{\prime}$.

Since every rewrite step $t_{i-1} \rightarrow_{R \cup E} t_{i}$ at position $\lambda$ corresponds to a rewrite step $t_{i-1}^{\prime} \rightarrow_{R \cup E}^{1}$ $t_{i}^{\prime}$, and there are infinitely many of such steps, it follows that the derivation $t_{n}^{\prime} \rightarrow{ }_{R \cup E}^{0,1}$ $t_{n+1}^{\prime} \rightarrow_{R \cup E}^{0,1} \cdots$ has infinitely many rewrite steps.

Note that all subterms at depth 1 in $t_{n}^{\prime}$ are reachable from constants. Therefore, there exists a ground flat term $t$ with height 1 such that $t \rightarrow_{R \cup E}^{*} t_{n}^{\prime}$ holds, and hence, there exists an infinite derivation from a ground flat term $t$. To finish the proof, we only need to prove that this infinite derivation has infinitely many rewrite steps with $R$.

We proceed by contradiction by assuming that $t_{n}^{\prime} \rightarrow_{R \cup E}^{0,1} t_{n+1}^{\prime} \rightarrow_{R \cup E}^{0,1} \ldots$ contains only finitely many rewrite steps with $R$. Hence, there exists an $N \geq n$ such that the derivation $t_{N}^{\prime} \rightarrow_{R \cup E}^{0,1} t_{N+1}^{\prime} \rightarrow_{R \cup E}^{0,1} \ldots$ contains no steps with $R$. Call this derivation $\pi^{\prime}$. We can observe the following properties about the corresponding old derivation $t_{N} \rightarrow_{R \cup E} t_{N+1} \rightarrow_{R \cup E} \ldots$, which we name $\pi$ :

(a) All rewrite steps at position $\lambda$ in the derivation $\pi$ are performed with $E$ : if there was a rewrite step $t_{i} \rightarrow_{R, \lambda} t_{i+1}$ in $\pi$, then we would have had $t_{i}^{\prime} \rightarrow_{R, \lambda}^{1} t_{i+1}^{\prime}$ in $\pi^{\prime}$, which contradicts the fact that there are no rewrite steps with $R$ in $\pi^{\prime}$.

(b) In $\pi$, there are infinitely many rewrite steps of the form $t_{i} \rightarrow_{R, j . p} t_{i+1}$ where $\left.t_{i}\right|_{j}$ is not reachable from a constant: we know that there are infinitely many rewrite steps with $R$ in $\pi$, but there are no rewrite steps with $R$ in $\pi^{\prime}$, and hence, all the (infinitely many) rewrite steps with $R$ in $\pi$ have to fall in Case (2) above.

From facts (a) and (b), it follows that there is a subterm $\left.t_{N}\right|_{j}$ that is not reachable from a constant and such that there is an infinite derivation starting from $\left.t_{N}\right|_{j}$ that uses infinitely many rewrites with $R$. This is because any subterm at depth 1 in the derivation $\pi$ that is not reachable from a constant is either (i) left unchanged by a rewrite step in $\pi$ (it may be moved to another position at depth 1), or (ii) it is rewritten into a subterm at depth 1 that is also not reachable from a constant (because of the choice of $n$ and the fact that $N \geq n$ ). A subterm that is reachable from a constant can not be rewritten into a term that is not reachable from a constant.

As before, the subterm $\left.t_{N}\right|_{j}$ is reachable from either a constant or a proper subterm of $t_{0}$. Hence, there is an infinite derivation with infinitely many rewrite steps with $R$ starting from a constant or a proper subterm of $t_{0}$, contradicting the minimality of $t_{0}$.

Now, the main result follows immediately from Lemmas 3.1, 3.2, 3.3, 6.3 and Theorem 4.10,

Theorem 6.4. Termination and innermost termination are both decidable for rewriting with right-shallow right-linear TRS modulo permutative theories.

\section{TERMINATION IS PSPACE-HARD FOR FLAT RIGHT-LINEAR TRS}

The algorithms of the previous sections decide termination by essentially generating all terms reachable from ground flat terms up to a height linearly bounded by the size of TRS $R$. Thus, these algorithms run in double exponential time, since there are that many 
different reachable terms to consider. In this section we give a lower bound for the time complexity of these problems.

Theorem 7.1. The termination and innermost termination are PSPACE-hard for flat right-linear TRS.

Proof. We reduce from the following automata intersection problem, which is well-known to be PSPACE-complete [Koz77, to non-termination:

Input: $n$ finite (word) automata $A_{1}, \ldots, A_{n}$.

Question: $\quad \mathcal{L}\left(A_{1}\right) \cap \ldots \cap \mathcal{L}\left(A_{n}\right) \neq \emptyset$ ?

Let $\left\langle Q_{1}, \Sigma, q_{01}, F_{1}, \Delta_{1}\right\rangle, \ldots,\left\langle Q_{n}, \Sigma, q_{0 n}, F_{n}, \Delta_{n}\right\rangle$ be $A_{1}, \ldots, A_{n}$, respectively, more explicitly written. Here $Q_{i}$ is the set of states, $\Sigma$ is the alphabet, $q_{0 i}$ is the initial state, $F_{i}$ is the set of final states and $\Delta_{i}$ is the set of transitions of the $i$-th automaton. Without loss of generality, we assume that $\Sigma$ is $\{a, b\}$.

Our goal is to construct a TRS $R$ satisfying that $R$ is non-terminating if and only if $\mathcal{L}\left(A_{1}\right) \cap \ldots \cap \mathcal{L}\left(A_{n}\right) \neq \emptyset$ holds. It is easy to check whether the empty word $\lambda$ is accepted by all $A_{i}$. In the affirmative case we may generate, as the result of our reduction, a trivially non-terminating TRS. Thus, from now on, assume that $\lambda$ is not in $\mathcal{L}\left(A_{1}\right) \cap \ldots \cap \mathcal{L}\left(A_{n}\right)$.

The idea behind the construction of $R$ is as follows. A word $w$, say $a b a$, is encoded by terms, either $f(a, f(b, a))$ or $f(f(a, b), a)$. We will include rules in $R$ so that if $w \in \mathcal{L}\left(A_{i}\right)$, then $c_{i}$ can $R$-reach every possible representation of $w$. If $\mathcal{L}\left(A_{1}\right) \cap \ldots \cap \mathcal{L}\left(A_{n}\right) \neq \emptyset$, then we would like to get a nonterminating derivation $c \rightarrow h\left(c_{1}, \ldots, c_{n}\right) \rightarrow h(t, \ldots, t) \rightarrow c \rightarrow \cdots$ using the rules $c \rightarrow h\left(c_{1}, \ldots, c_{n}\right)$ and $h(x, \ldots, x) \rightarrow c$ in $R$. To ensure that "all other rules" of $R$ are terminating, the constant $c_{i}$ will not reach all terms in $\mathcal{L}\left(A_{i}\right)$, but only terms of a bounded length.

Let $M$ be $\left|Q_{1}\right| \cdot\left|Q_{2}\right| \cdots\left|Q_{n}\right|$. Let $N$ be $\left\lceil\log _{2}(M)\right\rceil$. Formally, $R$ is defined over the following alphabet.

$$
\begin{aligned}
\bar{\Sigma} & =\bar{\Sigma}_{0} \cup \bar{\Sigma}_{2} \cup \bar{\Sigma}_{n} \\
\bar{\Sigma}_{0} & =\left\{a, b, c, c_{1}, \ldots, c_{n}\right\} \cup\left\{c_{i j q \hat{q}} \mid i \in\{1, \ldots, n\}, j \in\{0, \ldots, N\}, q, \hat{q} \in Q_{i}\right\} \\
\bar{\Sigma}_{2} & =\{f\} \\
\bar{\Sigma}_{n} & =\{h\}
\end{aligned}
$$

$R$ is defined to contain the following rules:

$$
\begin{array}{rlrl}
c & \rightarrow h\left(c_{1}, c_{2}, \ldots, c_{n}\right) & \\
h(x, x, \ldots, x) & \rightarrow c & & \\
c_{i} & \rightarrow c_{i 0 q_{0 i} q} & \left.i \in\{1, \ldots, n\}, q \in F_{i}\right\} \\
c_{i j q \hat{q}} & \rightarrow a & i \in\{1, \ldots, n\}, j \in\{0, \ldots, N\},(q a \rightarrow \hat{q}) \in \Delta_{i} \\
c_{i j q \hat{q}} & \rightarrow b & i \in\{1, \ldots, n\}, j \in, \ldots, N\},(q b \rightarrow \hat{q}) \in \Delta_{i} \\
c_{i j q \hat{q}} & \rightarrow f\left(c_{i(j+1) q \bar{q}}, c_{i(j+1) \bar{q} \hat{q}}\right) & i \in\{1, \ldots, n\}, j \in\{0, \ldots, N-1\}, q, \bar{q}, \hat{q} \in Q_{i}
\end{array}
$$

Now, we prove that $R$ is non-terminating if and only if $\mathcal{L}\left(A_{1}\right) \cap \ldots \cap \mathcal{L}\left(A_{n}\right) \neq \emptyset$ holds.

$\Leftarrow$ : Suppose that $\mathcal{L}\left(A_{1}\right) \cap \ldots \cap \mathcal{L}\left(A_{n}\right)$ is not empty. In this case, it is well-known that there exists a word $w \in \mathcal{L}\left(A_{1}\right) \cap \ldots \cap \mathcal{L}\left(A_{n}\right)$ with size bounded by $M$. Thus, there exists a term $t$ with height bounded by $N$, with $f$ in all its internal nodes, and whose sequence of leaves is $w$. It is clear that $c$ reaches $h(t, \ldots, t)$. Moreover, by using the rule $h(x, \ldots, x) \rightarrow c$, this term reaches $c$. Therefore, $c \rightarrow_{R}^{+} c$. Hence, $R$ is nonterminating.

$\Rightarrow$ : Suppose that $\mathcal{L}\left(A_{1}\right) \cap \ldots \cap \mathcal{L}\left(A_{n}\right)$ is empty. In order to prove termination of $R$, it suffices to prove termination of $R$ starting from any right-hand side term of $R$. Suppose $R$ does not terminate starting from the term $s$. 
(a) First, we observe that $R$ terminates from all constants of $\bar{\Sigma}_{0}-\{c\}$ independently of the form of $A_{1}, \ldots, A_{n}$. Hence, $s \notin \bar{\Sigma}_{0}-\{c\}$.

(b) Consider the case when $s$ is $c$. But, the fact that $\mathcal{L}\left(A_{1}\right) \cap \ldots \cap \mathcal{L}\left(A_{n}\right)$ is empty ensures that $R$ is also terminating from $c$, and hence $s \notin \bar{\Sigma}_{0}$.

(c) If $s$ is $h\left(c_{1}, \ldots, c_{n}\right)$, then either there is a derivation with infinitely many rewrite steps starting from some $c_{i}$ or there is a derivation with infinitely many rewrite steps and starting from $c$. We argued above that none of these cases is possible.

(d) If $s$ is $f\left(c_{i(j+1) q \bar{q}}, c_{i(j+1) \bar{q} \hat{q}}\right)$, then, since there is no rule with left-hand side rooted by $f$, there is a derivation with infinitely many rewrite steps starting from one of the arguments. We argued above that there are no derivations with infinitely many rewrite steps and starting from constants.

We finish the proof by noting that the size of $R$ is $O\left(n N \sum_{i=1}^{n}\left(\left|Q_{i}\right|^{3}+\left|\Delta_{i}\right|\right)\right)$, which is polynomial in the size $\sum_{i=1}^{n}\left(\left|Q_{i}\right|+\left|\Delta_{i}\right|\right)$ of the automata intersection problem.

\section{UNDECIDABILITY OF TERMINATION FOR FLAT TRS}

In this section, we prove undecidability of termination for flat TRS, and undecidability of innermost termination for right-flat TRS. This is done by a reduction from the Post correspondence problem $(\mathrm{PCP})$ restricted to nonempty strings, which is defined as:

Input: $n$ pairs of strings $\left\langle u_{1}, v_{1}\right\rangle, \ldots,\left\langle u_{n}, v_{n}\right\rangle$ s.t. $u_{i} \neq \lambda, v_{i} \neq \lambda$ for all $i$

Question: Does there exist $k>0$ and $i_{1}, \ldots, i_{k}$ s.t. $1 \leq i_{1} \leq n, \ldots, 1 \leq i_{k} \leq n$ and $\left(u_{i_{1}} \cdots u_{i_{k}}=v_{i_{1}} \cdots v_{i_{k}}\right)$ ?

Since decidability of termination for flat TRS is equivalent to decidability of termination for shallow TRS (Lemmas 3.2 and 3.3), we will prove undecidability of termination for shallow TRS. Since PCP is not decidable but it is semi-decidable, and non-termination is semi-decidable for shallow TRS (Theorem 4.10), we will reduce PCP to non-termination of shallow TRS: a reduction to just termination is not possible. The reduction is given in the proof of Theorem 8.1, but to provide an intuition, we first illustrate it via a small example.

Consider a PCP instance $\left\langle u_{1}, v_{1}\right\rangle, \ldots,\left\langle u_{n}, v_{n}\right\rangle$ over a signature $\Sigma$. The $j$ 'th symbol of $u_{i}$ and $v_{i}$, whenever it exists, is denoted by $u_{i, j}$ and $v_{i, j}$ respectively. For example, $\langle a a, a\rangle,\langle b, a b a\rangle$ is a PCP instance over $\Sigma=\{a, b\}$. It has a solution $1,2,1$ since $a a \cdot b \cdot a a=$ $a \cdot a b a \cdot a$. We want to define a rewrite system $R$ such that $R$ is non-terminating iff there is such a solution. Let $n=2$ be the number of rules in the PCP instance and let $L=3$ be the maximum size of a string in the PCP instance. We define $R$ over a signature $\Sigma^{\prime}$ where where

$$
\begin{aligned}
& \Sigma^{\prime}:=\Sigma_{0}^{\prime} \cup \Sigma_{1}^{\prime} \cup \Sigma_{2}^{\prime} \cup \Sigma_{6}^{\prime} \cup \Sigma_{8}^{\prime} \\
& \Sigma_{0}^{\prime}:=\left\{U, U^{\prime}, V, V^{\prime}, P, P^{\prime}, P^{\prime \prime}, A, A^{\prime}, A^{\prime \prime}\right\} \\
& \Sigma_{1}^{\prime}:=\{a, b\} \cup\left\{U_{i, j}, V_{i, j}, P_{i, j} \mid i \in\{1, \ldots, n\}, j \in\{1, \ldots, L\}\right\}, \\
& \Sigma_{2}^{\prime}:=\left\{f_{1}\right\}, \quad \Sigma_{6}^{\prime}:=\left\{f_{3}\right\}, \quad \Sigma_{8}^{\prime}:=\left\{f_{2}\right\}
\end{aligned}
$$

A potential solution, say $i_{1}, \ldots, i_{k}$, to the PCP instance is encoded by a pair of terms $\left(s_{u u}, s_{v v}\right)$ where

$$
\begin{aligned}
s_{u u} & :=U_{i_{1}, 1} \ldots U_{i_{1}, L} \ldots U_{i_{k}, 1} \ldots U_{i_{k}, L}(U) \\
s_{v v} & :=V_{i_{1}, 1} \ldots V_{i_{1}, L} \ldots V_{i_{k}, 1} \ldots V_{i_{k}, L}(V)
\end{aligned}
$$


Concretely, the solution $1,2,1$ is encoded by the pair

$$
\begin{aligned}
s_{u u} & :=U_{1,1} U_{1,2} U_{1,3} U_{2,1} U_{2,2} U_{2,3} U_{1,1} U_{1,2} U_{1,3}(U) \\
s_{v v} & :=V_{1,1} V_{1,2} V_{1,3} V_{2,1} V_{2,2} V_{2,3} V_{1,1} V_{1,2} V_{1,3}(V)
\end{aligned}
$$

Here the notation $U_{1,1} U_{1,2} U_{1,3}(U)$ serves as a shorthand for the term $U_{1,1}\left(U_{1,2}\left(U_{1,3}(U)\right)\right)$. This convention allows us to view strings as (parts of) terms. We need to construct a rewrite system $R$ whose non-termination implies that $s_{u u}, s_{v v}$ indeed correspond to a solution of the PCP. Hence, we need to check that

(1) $s_{u u}$ and $s_{v v}$ are of the form above,

(2) the indices sequence $i_{1}, \ldots, i_{k}$ in $s_{u u}$ and $s_{v v}$ are the same, and

(3) the words $u_{i_{1}} \ldots u_{i_{k}}$ and $v_{i_{1}} \ldots v_{i_{k}}$ are the same.

To check (1), we introduce the following rules in $R$ :

$$
\begin{aligned}
& R_{U}:=\left\{U_{i, 1} U_{i, 2} \cdots U_{i, L}(U) \rightarrow U^{\prime}, U_{i, 1} U_{i, 2} \cdots U_{i, L}\left(U^{\prime}\right) \rightarrow U^{\prime} \mid i \in\{1, \ldots, n\}\right\} \\
& R_{V}:=\left\{V_{i, 1} V_{i, 2} \cdots V_{i, L}(V) \rightarrow V^{\prime}, V_{i, 1} V_{i, 2} \cdots V_{i, L}\left(V^{\prime}\right) \rightarrow V^{\prime} \mid i \in\{1, \ldots, n\}\right\}
\end{aligned}
$$

We note that $s_{u u} \rightarrow_{R_{U}}^{*} U^{\prime}$ and $s_{v v} \rightarrow_{R_{V}}^{*} V^{\prime}$. Hence we can check (1) by checking if $s_{u u} \rightarrow_{R}^{*} U^{\prime}$ and $s_{v v} \rightarrow_{R}^{*} V^{\prime}$. But this does not still check that the sequence $i_{1}, \ldots, i_{k}$ (sequence $1,2,1$ in the example) used in $s_{u u}$ is the same as the one used in $s_{v v}$.

To check (2), we make $U_{i, j}(x)$ and $V_{i, j}(x)$ rewrite to $P_{i, j}(x)$. Hence, we introduce the following rules in $R$ :

$$
\begin{aligned}
R_{2 P}:=\left\{U_{i, j}(x) \rightarrow P_{i, j}(x), V_{i, j}(x) \rightarrow P_{i, j}(x) \mid i \in\{1, \ldots, n\}, j \in\{1, \ldots, L\}\right\} \\
R_{P^{\prime}}:=\left\{P_{i, 1} P_{i, 2} \cdots P_{i, L}\left(P^{\prime}\right) \rightarrow P^{\prime} \mid i \in\{1, \ldots, n\}\right\} \\
R_{P^{\prime \prime}}:=\left\{P_{i, 1} P_{i, 2} \cdots P_{i, L}\left(P^{\prime \prime}\right) \rightarrow P^{\prime \prime} \mid i \in\{1, \ldots, n\}\right\} \\
R_{U P}:=\left\{U \rightarrow P, V \rightarrow P, P \rightarrow P^{\prime}, P \rightarrow P^{\prime \prime}\right\}
\end{aligned}
$$

Now, using these new rules, we note that $s_{u u}$ and $s_{v v}$ are joinable if they use the same sequence of indices $i_{1}, \ldots, i_{k}$. In fact, both $s_{u u}$ and $s_{v v}$ rewrite to the term $s_{p p}$, where

$$
s_{p p}:=P_{i_{1}, 1} \cdots P_{i_{1}, L} \cdots P_{i_{k}, 1} \cdots P_{i_{k}, L}(P)
$$

Moreover, using $R_{P^{\prime \prime}} \cup R_{U P}, s_{p p}$ can rewrite to either $P^{\prime}$ or $P^{\prime \prime}$. Thus, we can check (2) by checking for the joinability of $s_{u u}$ and $s_{v v}$ to a term that can reach both $P^{\prime}$ and $P^{\prime \prime}$.

Finally, to check (3), we introduce the following rules in $R$ :

$$
\begin{aligned}
R_{U A}:= & \left\{U \rightarrow A, V \rightarrow A, A \rightarrow A^{\prime}, A \rightarrow A^{\prime \prime}\right\} \\
R_{\alpha}:= & \left\{\alpha\left(A^{\prime}\right) \rightarrow A^{\prime}, \alpha\left(A^{\prime \prime}\right) \rightarrow A^{\prime \prime} \mid \alpha \in \Sigma\right\} \\
R_{w}:= & \left\{U_{i, j}(x) \rightarrow u_{i, j}(x)|1 \leq j \leq| u_{i} \mid\right\} \cup\left\{U_{i, j}(x) \rightarrow x|j>| u_{i} \mid\right\} \\
& \cup\left\{V_{i, j}(x) \rightarrow v_{i, j}(x)|1 \leq j \leq| v_{i} \mid\right\} \cup\left\{V_{i, j}(x) \rightarrow x|j>| v_{i} \mid\right\}
\end{aligned}
$$

Using these rules, $s_{u u}$ and $s_{v v}$ can both rewrite to a common term $(w(A))$ if the strings $u_{i_{1}} \ldots u_{i_{k}}$ and $v_{i_{1}} \ldots v_{i_{k}}$ are equal (to $w$ ). In our example, $w:=$ aabaa. Moreover, the common reachable term $(w(A))$ can then rewrite to either $A^{\prime}$ or $A^{\prime \prime}$. Hence, we can check $(3)$ by checking for joinability of $s_{u u}$ and $s_{v v}$ to a term that can reach both $A^{\prime}$ and $A^{\prime \prime}$.

We can put everything together by introducing three more rules in $R$ :

$$
\begin{aligned}
R_{f}:= & \left\{f_{1}(x, y) \rightarrow f_{2}(x, y, x, y, x, y, x, y), f_{2}\left(x, y, z, z, z^{\prime}, z^{\prime}, U^{\prime}, V^{\prime}\right) \rightarrow f_{3}\left(x, y, z, z, z^{\prime}, z^{\prime}\right),\right. \\
& \left.f_{3}\left(x, y, A^{\prime}, A^{\prime \prime}, P^{\prime}, P^{\prime \prime}\right) \rightarrow f_{1}(x, y)\right\}
\end{aligned}
$$


If $s_{u u}, s_{v v}$ is generated from a solution of the PCP instance, then we can immediately get a nonterminating derivation using $R$ :

$$
\begin{aligned}
& f_{1}\left(s_{u u}, s_{v v}\right) \rightarrow R_{f} \quad f_{2}\left(s_{u u}, s_{v v}, s_{u u}, s_{v v}, s_{u u}, s_{v v}, s_{u u}, s_{v v}\right) \\
& \rightarrow_{R_{U}, R_{V}}^{*} \quad f_{2}\left(s_{u u}, s_{v v}, s_{u u}, s_{v v}, s_{u u}, s_{v v}, U^{\prime}, V^{\prime}\right) \\
& \rightarrow_{R_{2 P}, R_{U P}}^{*} \quad f_{2}\left(s_{u u}, s_{v v}, s_{u u}, s_{v v}, s_{p p}, s_{p p}, U^{\prime}, V^{\prime}\right) \\
& \rightarrow_{R_{w}, R_{U A}}^{*} \quad f_{2}\left(s_{u u}, s_{v v}, w(A), w(A), s_{p p}, s_{p p}, U^{\prime}, V^{\prime}\right) \\
& \rightarrow_{R_{f}} \quad f_{3}\left(s_{u u}, s_{v v}, w(A), w(A), s_{p p}, s_{p p}\right) \\
& \rightarrow R_{P^{\prime}, R_{P^{\prime \prime}}, R_{U P}}^{*} f_{3}\left(s_{u u}, s_{v v}, w(A), w(A), P^{\prime}, P^{\prime \prime}\right) \\
& \rightarrow_{R_{\alpha}, R_{U A}}^{*} \quad f_{3}\left(s_{u u}, s_{v v}, A^{\prime}, A^{\prime \prime}, P^{\prime}, P^{\prime \prime}\right) \\
& \rightarrow R_{f} \quad f_{1}\left(s_{u u}, s_{v v}\right)
\end{aligned}
$$

The following theorem formally describes and proves this reduction.

Theorem 8.1. Termination of shallow TRS is undecidable.

Proof. Consider an instance $\left\langle u_{1}, v_{1}\right\rangle, \ldots,\left\langle u_{n}, v_{n}\right\rangle$ of the restricted PCP, that is, $u_{i}, v_{i}$ are nonempty strings over alphabet $\Sigma$. We construct a shallow TRS $R$ such that this PCP instance has a solution iff $R$ is non-terminating.

Let $L=\operatorname{Max}\left(\left|u_{1}\right|, \ldots,\left|u_{n}\right|,\left|v_{1}\right|, \ldots,\left|v_{n}\right|\right)$. We construct $R$ over a signature $\Sigma^{\prime}$, where $\Sigma^{\prime}$ is defined in Equation 8.1. The TRS $R$ is defined as follows:

$$
R:=R_{U} \cup R_{V} \cup R_{2 P} \cup R_{P^{\prime}} \cup R_{P^{\prime \prime}} \cup R_{U P} \cup \cup R_{\alpha} \cup R_{w} \cup R_{U A} \cup R_{f}
$$

where $R_{U}, R_{V}$ are defined in Equation 8.3, $R_{2 P}, R_{P^{\prime}}, R_{P^{\prime \prime}}, R_{U P}$ are defined in Equation 8.4. $R_{\alpha}, R_{w}, R_{U A}$ are defined in Equation 8.5 and $R_{f}$ is defined in Equation 8.6.

$\Rightarrow$ : We first show that if the PCP instance has a solution, then $R$ is non-terminating. Let $i_{1}, \ldots, i_{k}$ be a solution of the PCP instance, i.e. $w=u_{i_{1}} \ldots u_{i_{k}}=v_{i_{1}} \ldots v_{i_{k}}$ holds. Then, we have the infinite derivation, shown in Equation 8.7, starting from the ground term $s_{1}:=f_{1}\left(s_{u u}, s_{v v}\right)$, where $s_{u u}$ and $s_{v v}$ are defined in Equation 8.2

$\Leftarrow$ : Suppose $R$ does not terminate. We need to show that the PCP instance has a solution. To this end we define the concept of $U V$-variant. We say that a term $s$ is a $U V$-variant of a term $t$, if $t$ can be obtained from $s$ by applying several rewrite steps using rules from the subset $\left\{U_{i, j}(x) \rightarrow x: i=1 \ldots n, j>\left|u_{i}\right|\right\} \cup\left\{V_{i, j}(x) \rightarrow x: i=1 \ldots n, j>\left|v_{i}\right|\right\}$ of $R_{w}$. Note that, since none of $u_{i}$ or $v_{i}$ is $\lambda$ in the original PCP instance, $s$ and $t$ have the same number of occurrences of symbols of $\left\{U_{i, 1}: i=1 \ldots n\right\} \cup\left\{V_{i, 1}: i=1 \ldots n\right\}$.

Now, note that since all rules in $R$ are height-preserving or height-decreasing, there is a derivation with infinitely many rewrite steps at the top. We pick such a derivation, but with minimal height for the initial term $t$. Then, the root symbol of $t$ has to be one of the $f_{i}$ 's: otherwise, only a finite number of rewrite steps can be done at the top and preserving the height. Therefore, we have a derivation of the form $f_{1}(\ldots) \rightarrow^{*} f_{2}(\ldots) \rightarrow^{*} f_{3}(\ldots) \rightarrow^{*}$ $f_{1}(\ldots) \rightarrow^{*} \ldots$ with infinitely many rewrite steps at the top. We can assume that we start with a term of the form $f_{1}(u, v)$. By observing the $R_{f}$ rules, one can deduce that $u$ and $v$ reach $A^{\prime}, A^{\prime \prime}, P^{\prime}, P^{\prime \prime}$, and that $u$ reaches $U^{\prime}$ and that $v$ reaches $V^{\prime}$. This is possible only if the terms $u$ and $v$ are $U V$-variants of terms of the form

$$
\begin{aligned}
s_{u u} & :=U_{i_{1}, 1} \ldots U_{i_{1}, L} \ldots U_{i_{k}, 1} \ldots U_{i_{k}, L}(U) \\
s_{v v} & :=V_{j_{1}, 1} \ldots V_{j_{1}, L} \ldots V_{j_{k^{\prime}}, 1} \ldots V_{j_{k^{\prime}}, L}(V)
\end{aligned}
$$


where $k, k^{\prime} \geq 1$. But, moreover, these terms have to be joinable to a term of the form $P_{i_{1}, 1} \ldots P_{i_{1}, L} \ldots P_{i_{k}, 1} \ldots P_{i_{k}, L}(P)$, and also of the form $P_{j_{1}, 1} \ldots P_{j_{1}, L} \ldots P_{j_{k^{\prime}}, 1} \ldots P_{j_{k^{\prime}}, L}(P)$. (Note here that since $u_{i}, v_{i}$ are not $\lambda$, terms like $U_{i, 1} \ldots U_{i, L}(x)$ can not rewrite to $x$ and hence the indices $i_{1}, \ldots, i_{k}, j_{1}, \ldots, j_{k^{\prime}}$ will be preserved in any joinability proof.) Hence, $k=k^{\prime}$ and $i_{r}=j_{r}$ for all $r$ in $\{1, \ldots, k\}$. But moreover, $u$ and $v$ have to be joinable to a term of the form $u_{i_{1}} \ldots u_{i_{k}}(A)=v_{i_{1}} \ldots v_{i_{k}}(A)$. Hence, $u_{i_{1}} \ldots u_{i_{k}}=v_{i_{1}} \ldots v_{i_{k}}$ and there is a solution of the original PCP.

Remark: It is important to keep $P^{\prime}$ and $P^{\prime \prime}$ (and $A^{\prime}$ and $A^{\prime \prime}$ ) as two different constants in the above proof. If we reuse $P$ in place of $P^{\prime}$ and $P^{\prime \prime}$ (respectively, $A$ in place of $A^{\prime}$ and $A^{\prime \prime}$ ), then terms that satisfy Check (1), but do not satisfy Check (2) (respectively, Check (3)), such as, $s_{u u}:=U_{1,1} U_{1,2} U_{1,3}(U)$ and $s_{v v}:=V_{2,1} V_{2,2} V_{2,3}(V)$, which do not correspond to a solution of the PCP, would generate infinite derivations starting from $f\left(s_{u u}, s_{v v}\right)$.

Combining Theorem 8.1 with Lemmas 3.2 and 3.3 , we have the following result.

Theorem 8.2. Termination of flat TRS is undecidable.

For the case of innermost rewriting, we have seen that termination is decidable for flat TRS. However, in the innermost case we have the following result.

Theorem 8.3. Innermost termination of right-flat TRS is undecidable.

Proof. Given an instance $\left\langle u_{i}, v_{i}\right\rangle, \ldots,\left\langle u_{n}, v_{n}\right\rangle$ of Post correspondence problem, we generate the TRS $R=\left\{f(x) \rightarrow g(x, x, x), g\left(x, u_{i}(y), v_{i}(z)\right) \rightarrow h(x, y, z), h\left(x, u_{i}(y), v_{i}(z)\right) \rightarrow\right.$ $h(x, y, z), h(x, \epsilon, \epsilon) \rightarrow f(x) \mid 1 \leq i \leq n\}$. Here $\epsilon$ is a constant representing the empty string. Note that $R$ is right-flat. It is easy to see that the PCP instance has a solution iff $R$ is innermost non-terminating.

Remark: A reduction similar to the one in the proof of Theorem 8.3 was given in Definition 5.3.6 of BKdV03 for showing undecidability of termination for (general) term rewriting systems.

\section{Conclusions}

We showed that termination and innermost termination of right-shallow right-linear term rewriting systems is decidable. This result also holds when we consider rewriting modulo permutative theories. We also showed that innermost termination of flat TRSs is decidable, whereas termination of flat TRSs is undecidable. For the decidable problems, the complexity of the given algorithms is doubly exponential, whereas we have also provided a PSPACE-hardness lower bound. It is unclear whether both upper and lower bounds can be improved in some way. As further work it would be interesting to fix the exact complexity of these problems, but also to consider other classes of TRS, for example, classes defined by imposing syntactic restrictions not on the original TRS, but on the dependency pairs of the TRS [WS06, USS10].

\section{REFERENCES}

[BKdV03] M. Bezem, J. W. Klop, and R. de Vrijer, editors. Term Rewriting Systems. Cambridge Tracts in Theoretical Computer Science 55. Cambridge University Press, 2003.

[BN98] F. Baader and T. Nipkow. Term Rewriting and All That. Cambridge University Press, New York, 1998. 
[Der81] N. Dershowitz. Termination of linear rewriting systems. In Proc. 8th Colloquium on Automata, Languages and Programming, ICALP, volume 115 of LNCS, pages 448-458, 1981.

[GHT07] G. Godoy, E. Huntingford, and A. Tiwari. Termination of rewriting with right-flat rules. In Proc. 18th Intl. Conf. on Rewriting Techniques and Applications, RTA, volume 4533 of LNCS, pages 200-213, 2007.

[GT04] G. Godoy and A. Tiwari. Deciding fundamental properties of right-(ground or variable) rewrite systems by rewrite closure. In Proc. Intl. Joint Conf. on Automated Deduction, IJCAR, volume 3097 of LNAI, pages 91-106. Springer, July 2004.

[GT05] G. Godoy and A. Tiwari. Termination of rewrite systems with shallow right-linear, collapsing, and right-ground rules. In Proc. 20th Intl. Conf. on Automated Deduction, CADE, volume 3632 of $L N C S$, pages 164-176. Springer, July 2005.

[HL78] G. Huet and D. S. Lankford. On the uniform halting problem for term rewriting systems. INRIA, Le Chesnay, France, 1978. Technical Report 283.

[Koz77] D. Kozen. Lower bounds for natural proof systems. In Proc. 18th Symp. on the Foundations of Computer Science, pages 254-266, 1977.

[Pla93] D. A. Plaisted. Polynomial time termination and constraint satisfaction tests. In Proc. 5th Intl. Conf. on Rewriting Techniques and Applications, RTA, volume 690 of LNCS, pages 405-420, 1993.

[TKS00] T. Takai, Y. Kaji, and H. Seki. Right-linear finite path overlapping term rewriting systems effectively preserve recognizability. In Proc. 11th Intl. Conf. on Rewriting Techniques and Applications, RTA, volume 1833 of $L N C S$, pages 246-260, 2000.

[Toy87] Y. Toyama. Counterexamples to termination for the direct sum of term rewriting systems. Information Processing Letters, 25:141-143, 1987.

[USS10] K. Uchiyama, M. Sakai, and T. Sakabe. Decidability of termination and innermost termination for term rewriting systems with right-shallow dependency pairs. IEICE Trans. on Information and Systems, E93-D(5):953-962, 2010.

[WS06] Y. Wang and M. Sakai. Decidability of termination for semi-constructor trss, left-linear shallow trss and related systems. In Proc. 17th Intl. Conf. on Rewriting Techniques and Applications, RTA, volume 4098 of $L N C S$, pages 343-356. Springer, 2006. 